\title{
WEIGHTED KNEADING THEORY OF ONE-DIMENSIONAL MAPS WITH A HOLE
}

\author{
J. LEONEL ROCHA and J. SOUSA RAMOS
}

Received 30 April 2003

\begin{abstract}
The purpose of this paper is to present a weighted kneading theory for one-dimensional maps with a hole. We consider extensions of the kneading theory of Milnor and Thurston to expanding discontinuous maps with a hole and introduce weights in the formal power series. This method allows us to derive techniques to compute explicitly the topological entropy, the Hausdorff dimension, and the escape rate.
\end{abstract}

2000 Mathematics Subject Classification: 37E05, 37C30, 37B10, 37C45.

1. Introduction. Let $I \subset \mathbb{R}$ be a compact interval and $F$ a linear expanding map on $I$. We study the dynamical system given by iterating points by $F$. Of particular interest is the set defined by the points that remain in the domain of $F$ under iteration. An equivalent way of viewing this situation is as the inverse of an iterated function system (IFS) (see [4]). Let $f=\left\{f_{i}\right\}_{i=1}^{n}$ be an IFS, a collection of self-maps on $I$, defined by

$$
f_{i}(x):=\rho_{i} x+\varrho_{i}, \quad i=1, \ldots, n,
$$

where for all $i, 0<\left|\rho_{i}\right|<1$ and $\varrho_{i} \in \mathbb{R}$. Let $E$ be the corresponding self-similar set, the attractor. If $f_{i}$ is monotone, then it is usual to see $E$ as the repeller of a linear expanding map $F: \bigcup_{i=1}^{n} f_{i}(I) \rightarrow I$, which will be denoted by $F=\left(F_{1}, \ldots, F_{n}\right)$, where

$$
F_{i}(x):=f_{i}^{-1}(x) \text { if } x \in f_{i}(I)
$$

We consider the piecewise linear map $F$ with a single hole, that is, there is an open subinterval $I_{h} \subset I$ with $I_{h} \neq \varnothing$ such that $I$ is the disjoint union of $I_{h}$ and $\bigcup_{i=1}^{n} \operatorname{Im}\left(f_{i}\right)$ (see $[8,10])$. The points $x \in I_{h}$ will be mapped out of $I$ and the same will happen to all the points $x \in F^{-k}\left(I_{h}\right)$ for $k \geq 1$. The set $\bigcup_{k} F^{-k}\left(I_{h}\right)$ is open and dense in $I$ and has full Lebesgue measure (see [14]).

The hole and the set of $n$ laps of $F$ determines a partition $\mathscr{P}_{I}:=\left\{I_{1}, \ldots, I_{h}, \ldots, I_{n}\right\}$ of the interval $I$. Considering the orbits of the lateral limit points of the discontinuity points and turning points, we define a Markov partition $\mathscr{P}_{I}^{\prime}$ of $I$.

The outline of the paper is as follows. In Section 2, we develop a weighted kneading theory to expanding discontinuous maps with a hole. In this section, we give a brief presentation of the kneading theory associated to $F$. For more details, see [6], and for maps of the interval with holes, see [9]. The new kneading approach using weights is inspired on [8], where we compute explicitly the escape rate that is characterized by a conditional invariant measure. The weights introduced in the kneading theory are 
defined by the inverses of the derivatives of the iterates of the discontinuity points and turning points of $F$, associated to a real parameter $\beta$. We consider the transfer or Perron-Frobenius operator $L_{\phi}$ associated to the map $F$ and to the Markov partition $\mathscr{P}_{I}^{\prime}$. The transfer operator has a matrix representation, which we will denote by $Q_{\beta}$. This matrix can be viewed as the matrix of $L_{\phi}$ acting on a finite-dimensional vector space of functions.

It is known that the spectrum of the transfer operator determines the ergodicity of the dynamical system. In Section 3, we will give an algorithm to compute this spectrum and to relate the transfer operator with the weighted kneading determinant $D(t, \beta)$ (see Theorem 3.6). To establish this relation, we introduce a weighted matrix $V_{\beta}$ and we use complexes and homology with weights (see Theorem 3.5). These results allow us to prove the main result.

THEOREM 1.1. Suppose that the kneading data associated to an expanding discontinuous map with a hole F correspond to periodic, eventually periodic orbits or to orbits that lie in the hole. Let $D(t, \beta)$ be the weighted kneading determinant.

(i) If $\beta$ is the unique solution of $D(1, \beta)=0$, then $\beta$ is the Hausdorff dimension of the attractor $E$.

(ii) If $t_{1}$ is the least real positive solution of $D(t, 1)=0$, then $\log \left(t_{1}\right)$ is the escape rate of the pair $(E, F)$.

(iii) If $t_{0}$ is the least real positive solution of $D(t, 0)=0$, then $\log \left(t_{0}^{-1}\right)$ is the topological entropy of the map $F$.

We remark that we can obtain the same results for a finite union of disjoint holes $I_{h_{j}} \subset I$.

2. Weighted kneading theory and subshifts of finite type. Let $a_{i}$, with $i=1, \ldots, n+1$, be the endpoints of the intervals in the partition $\mathscr{P}_{I}$. These points correspond to the discontinuity points and the turning points of the map $F$. We denote the hole by $I_{h}=$ $\left(a_{h}, a_{h+1}\right)$. Set

$$
\begin{aligned}
& \left\{a_{1}^{+}, a_{2}^{-}, a_{2}^{+}, \ldots, a_{h}^{-}, a_{h}^{+}, a_{h+1}^{-}, a_{h+1}^{+}, \ldots, a_{n+1}^{-}\right\} \\
& \quad=\left\{x^{(1)}, x^{(2)}, x^{(3)}, \ldots, x^{(2 h-2)}, x^{(2 h-1)}, x^{(2(h+1)-2)}, x^{(2(h+1)-1)}, \ldots, x^{(2 n)}\right\} .
\end{aligned}
$$

The orbit of each point $x^{(j)}$, with $j=1,2, \ldots, 2 h-2,2(h+1)-1, \ldots, 2 n$, is defined by

$$
o\left(x^{(j)}\right):=\left\{x_{k}^{(j)}: x_{k}^{(j)}=F^{k}\left(x^{(j)}\right), k \in \mathbb{N}_{0}\right\} .
$$

Concerning the itinerary of each point $x^{(j)}$, we will have

$$
F^{k}\left(x^{(j)}\right)=x_{k}^{(j)} \quad \text { or } \quad F^{k}\left(x^{(j)}\right) \in \operatorname{int}\left(I_{h}\right)
$$

In the first case, we have periodic, eventually periodic, or aperiodic orbits. While in the second, after a finite number of iterates, the itinerary of the points lies in the hole.

To simplify the presentation, we consider the points $x^{(1)}$ and $x^{(2 n)}$ as fixed points. To the orbit of each point $x^{(j)}$, with $j=2, \ldots, 2 h-2,2(h+1)-1, \ldots, 2 n-1$, we associate 
a sequence of symbols $S^{(j)}$ given by

$$
S^{(j)}:=S_{0}^{(j)} S_{1}^{(j)} \cdots S_{k}^{(j)} \cdots,
$$

where

$$
S_{k}^{(j)}:= \begin{cases}L & \text { if } F^{k}\left(x^{(j)}\right) \in I_{1}, \\ M_{s_{1}} & \text { if } F^{k}\left(x^{(j)}\right) \in I_{s_{1}+1}, s_{1} \in\{1, \ldots, h-2\}, \\ H & \text { if } F^{k}\left(x^{(j)}\right) \in I_{h}, \\ M_{S_{2}} & \text { if } F^{k}\left(x^{(j)}\right) \in I_{s_{2}+1}, s_{2} \in\{h-1, \ldots, n-2\}, \\ R & \text { if } F^{k}\left(x^{(j)}\right) \in I_{n} .\end{cases}
$$

We denote by $\mathscr{A}$ the ordered set of $n+1$ symbols, corresponding to the laps and the hole of $F$, that is,

$$
\mathscr{A}=\left\{L, M_{1}, \ldots, M_{h-2}, H, M_{h-1}, \ldots, M_{n-2}, R\right\}
$$

and according to the real-line order,

$$
L \prec M_{1} \prec \cdots \prec M_{h-2} \prec H \prec M_{h-1} \prec \cdots \prec M_{n-2} \prec R \text {. }
$$

We designate by $\mathscr{A}^{\mathbb{N}}$ the space of all sequences of symbols on the alphabet $\mathscr{A}$.

DEFINITION 2.1. The kneading data for the map $F$ is the (2n-4)-tuple of symbolic sequences

$$
\left(S^{(2)}, \ldots, S^{(2 h-2)}, S^{(2(h+1)-1)}, \ldots, S^{(2 n-1)}\right) \in \mathscr{A}^{\mathbb{N}} \times \mathscr{A}^{\mathbb{N}} \times \cdots \times \mathscr{A}^{\mathbb{N}} .
$$

The kneading increments introduced in [6] are defined by formal power series with coefficients in $\mathbb{Z}[[t]]$, the subring of the ring $\mathbb{Q}[[t]]$. For maps of the interval with a hole and more than one discontinuity point and turning points, we have several kneading increments, whose number depends on the number of discontinuity points and turning points of the map $F$ (see [9]). The kneading increments are defined by

$$
v_{a_{i}}(t):=\theta_{a_{i}^{+}}(t)-\theta_{a_{i}^{-}}(t)
$$

In the case where $a_{i}$ is an endpoint of the hole $I_{h}$, the increments are defined by

$$
v_{a_{h}}(t):=\theta_{a_{h}^{-}}(t), \quad v_{a_{h+1}}(t):=\theta_{a_{h+1}^{+}}(t),
$$

where $\theta_{a_{i}}(t)$ is the invariant coordinate of each symbolic sequence associated to the itinerary of each point $a_{i}$, with $1<i<n+1$. Each lateral invariant coordinate is defined by

$$
\theta_{a_{i}^{ \pm}}(t):=\lim _{x \rightarrow a_{i}^{ \pm}} \theta_{x}(t)=\sum_{k=0}^{\infty} \tau_{k} t^{k} S_{k}^{(j)}
$$


where $\tau_{0}:=1, \tau_{k}:=\prod_{l=0}^{k-1} \varepsilon\left(S_{l}^{(j)}\right), k>0$, with

$$
\varepsilon\left(S_{l}^{(j)}\right):= \begin{cases}1 & \text { if } F^{\prime}\left(F^{l}\left(a_{i}^{ \pm}\right)\right)>0, \\ 0 & \text { if } F^{\prime}\left(F^{l}\left(a_{i}^{ \pm}\right)\right) \in I_{h}, \\ -1 & \text { if } F^{\prime}\left(F^{l}\left(a_{i}^{ \pm}\right)\right)<0,\end{cases}
$$

and $S_{k}^{(j)}$ is the sequence of symbols corresponding to the orbits of $a_{i}^{ \pm}$.

The increments $v_{a_{i}}(t)$, with $1<i<n+1$, can also be written in the following way:

$$
v_{a_{i}}(t)=N_{i 1}(t) L+N_{i 2}(t) M_{1}+\cdots+N_{i(n-1)}(t) M_{n-2}+N_{i n}(t) R
$$

where the coefficients $N_{i j}(t) \in \mathbb{Z}[[t]]$ are the entries of an $n \times n$ matrix $N=\left[N_{i j}(t)\right]$. This matrix is called the kneading matrix associated to the map $F$. The kneading determinant is denoted by $D(t)$.

Now we are going to present the main definition of this paper, that is, the characterization of the weighted invariant coordinates. This definition allows us to construct a weighted kneading theory similar to the previous one.

DEFINITION 2.2. For the kneading data of the map $F$, the weighted invariant coordinate of each point $a_{i}$, with $1<i<n+1$ and $\beta \in \mathbb{R}$, is defined by

$$
\theta_{a_{i}^{ \pm}}(t, \beta):=\lim _{x \rightarrow a_{i}^{ \pm}} \theta_{x}(t, \beta)=\sum_{k=0}^{\infty} \tau_{k}\left(a_{i}^{ \pm}\right) t^{k} S_{k}^{(j)},
$$

where $\tau_{0}\left(a_{i}^{ \pm}\right):=1$ and for $k>0$,

$$
\boldsymbol{\tau}_{k}\left(a_{i}^{ \pm}\right):=\prod_{l=0}^{k-1} \varepsilon\left(S_{l}^{(j)}\right)\left|F^{\prime}\left(F^{l}\left(a_{i}^{ \pm}\right)\right)\right|^{-\beta} .
$$

Note that the derivative of the map $F$ satisfies the condition inf $\left|F_{i}^{\prime}(x)\right|>1$ on each interval $f_{i}(I)$. For each point $a_{i}$, with $i=1, \ldots, h-1, h+2, \ldots, n+1$, the weighted kneading increment is defined by

$$
v_{a_{i}}(t, \beta):=\theta_{a_{i}^{+}}(t, \beta)-\theta_{a_{i}^{-}}(t, \beta) .
$$

For the endpoints of the hole $I_{h}$, the weighted increments are defined by

$$
v_{a_{h}}(t, \beta):=\theta_{a_{h}^{-}}(t, \beta), \quad v_{a_{h+1}}(t, \beta):=\theta_{a_{h+1}^{+}}(t, \beta) .
$$

Separating the terms associated to the symbols on the alphabet $\mathscr{A}$, the weighted increments $v_{a_{i}}(t, \beta)$ are written in the following way:

$$
v_{a_{i}}(t, \beta)=N_{i 1}(t, \beta) L+N_{i 2}(t, \beta) M_{1}+\cdots+N_{i(n-1)}(t, \beta) M_{n-2}+N_{i n}(t, \beta) R .
$$

The coefficients $N_{i j}(t, \beta) \in \mathbb{R}[[t, \beta]]$ are the entries of an $n \times n$ matrix

$$
N(t, \beta):=\left[N_{i j}(t, \beta)\right],
$$


which we will call the weighted kneading matrix associated to $F$. The determinant of this matrix will be called the weighted kneading determinant and will be denoted by $D(t, \beta)$.

REMARK 2.3. To an eventually periodic orbit of a point $x^{(j)}$ represented by

$$
S_{0}^{(j)} \cdots S_{p-1}^{(j)}\left(S_{p}^{(j)} \cdots S_{p+q-1}^{(j)}\right)^{\infty}
$$

corresponds the weighted cyclotomic polynomial

$$
1-\prod_{l=0}^{q-1} \varepsilon\left(S_{p+l}^{(j)}\right)\left|F^{\prime}\left(F^{p+l}\left(x^{(j)}\right)\right)\right|^{-\beta} t^{q},
$$

where $q$ is the period of the orbit. If the orbit is periodic, then the weighted cyclotomic polynomial is

$$
1-\tau_{q}\left(x^{(j)}\right) t^{q}
$$

Now, let

$$
\left\{b_{1}, \ldots, b_{m+1}\right\}:=\left\{o\left(x^{(j)}\right): j=1, \ldots, 2 h-2,2(h+1)-1, \ldots, 2 n\right\}
$$

be the set of the points correspondent to the orbits of the lateral limit points of the discontinuity points and turning points ordered on the interval $I$. This set allows us to define a subpartition $\mathscr{P}_{I}^{\prime}$ of $\mathscr{P}_{I}=\left\{I_{1}, \ldots, I_{h}, \ldots, I_{n}\right\}$. The subpartition

$$
\mathscr{P}_{I}^{\prime}:=\left\{J_{1}, \ldots, J_{m}\right\},
$$

with $m \geq n$, determines a Markov partition of the interval $I$. Note that the hole is an element of the Markov partition. Note also that $F$ determines $\mathscr{P}_{I}^{\prime}$ uniquely, but the converse is not true.

The IFS $f$ induces a subshift of finite type whose $m \times m$ transition matrix $A=\left[a_{i j}\right]$ is defined by

$$
a_{i j}:= \begin{cases}1 & \text { if } F\left(\operatorname{int} J_{j}\right) \supseteq \operatorname{int} J_{i} \\ 0 & \text { otherwise. }\end{cases}
$$

We remark that if there exist $k$ points $b_{i}$ such that $b_{i} \in \operatorname{int} I_{h}$, with $1<i<m+1$, then the matrix $A$ has $k+1$ columns with all elements equal to zero, correspondent to the hole.

We denote this subshift by $\left(\Sigma_{A}, \sigma\right)$, where $\sigma$ is the shift map on $\Sigma_{m}^{\mathbb{N}}$ defined by $\sigma\left(x_{1} x_{2} \cdots\right):=x_{2} x_{3} \cdots$, with $\Sigma_{m}:=\{1, \ldots, m\}$ correspondent to the $m$ states of the subshift. Concerning this subshift $\left(\Sigma_{A}, \sigma\right)$ and the associated Markov partition $\mathscr{P}_{I}^{\prime}$, we consider a Lipschitz function $\phi: I \rightarrow \mathbb{R}$ defined by

$$
\phi:=\left\{\phi_{i}: J_{i} \longrightarrow \mathbb{R}, 1 \leq i \leq m\right\},
$$


where

$$
\phi_{i}(x):=-\beta \varphi_{i}(x), \quad \varphi_{i}(x):=\log \left|F_{i}^{\prime}(x)\right|, \quad \text { with } \beta \in \mathbb{R} .
$$

This function is a weight for the dynamical system associated to the subshift, depending on the real parameter $\beta$ (compare with [11]).

Let $\mathscr{L}^{1}(I)$ be the set of all Lebesgue integrable functions on $I$. The transfer operator $L_{\phi}: \mathscr{L}^{1}(I) \rightarrow \mathscr{L}^{1}(I)$, associated with $F$ and $\mathscr{P}_{I}$, is defined by

$$
\begin{aligned}
\left(L_{\phi} g\right)(x) & :=\sum_{j=1}^{n} \exp \phi\left(F_{j}^{-1}(x)\right) g\left(F_{j}^{-1}(x)\right) \chi_{F\left(\operatorname{int} I_{j}\right)} \\
& =\sum_{y: F y=x} \exp \phi(y) g(y),
\end{aligned}
$$

where $\chi_{I_{j}}$ is the characteristic function of $I_{j}$. Note that by definition of $F, F_{j}^{-1}(x)=$ $f_{j}(x)$, with $x \in F\left(I_{j}\right)$. Note also that, for any Borel subset $J \subset I$, we have

$$
F^{-1}(J)=\bigcup_{j=1}^{n} f_{j}\left(F\left(I_{j}\right) \cap J\right),
$$

where the sets $\left\{f_{j}\left(F\left(I_{j}\right) \cap J\right)\right\}_{j=1}^{n}$ are mutually disjoint. Depending on $J$, the set $f_{j}\left(F\left(I_{j}\right) \cap J\right)$ can be empty.

Now, we will restrict our attention to the transfer operator associated with $F$ and to the Markov partition $\mathscr{P}_{I}^{\prime}$. Given $J_{i} \in \mathscr{P}_{I}^{\prime}$, let $Y_{1 i}, \ldots, Y_{k i}$ be the preimages of $J_{i}$ under $F$, that is,

$$
F_{j}\left(\operatorname{int} Y_{j i}\right)=\operatorname{int} J_{i}, \quad \text { with } 1 \leq j \leq k, k \leq m .
$$

Then, we can define continuous maps $\left.f_{j}\right|_{J_{i}}:=\Psi_{j i}: J_{i} \rightarrow Y_{j i}$ that correspond to the IFS $f$ restricted to the interval $J_{i}$ such that $y_{j}=\Psi_{j i}(x)$ are the preimages of $x \in J_{i}$. Thus, for each $x \in J_{i}$, we have

$$
\left(L_{\phi_{j}} g\right)(x)=\sum_{j=1}^{k} \exp \phi_{j}\left(\Psi_{j i}(x)\right) g\left(\Psi_{j i}(x)\right) \delta\left(\Psi_{j i}(x)\right),
$$

where

$$
\delta\left(\Psi_{j i}(x)\right):= \begin{cases}1 & \text { if } \Psi_{j i}(x)=y_{j} \\ 0 & \text { otherwise }\end{cases}
$$

Nevertheless, for each interval $J_{j} \in \mathscr{P}_{I}^{\prime}$, we consider

$$
f_{j}(x):=\left.F^{-1}\right|_{J_{j}}(x) \quad \text { for } x \in F\left(\operatorname{int} J_{j}\right)=\bigcup_{a_{i j} \neq 0} \operatorname{int} J_{i}
$$


where $a_{i j}$ are the entries of the transition matrix $A$, with $1 \leq i, j \leq m$. By formula (2.31), we can write

$$
\left(L_{\phi_{j}} g\right)(x)=\sum_{j=1}^{m} \exp \phi_{j}\left(f_{j}(x)\right) g\left(f_{j}(x)\right) \chi_{F\left(\operatorname{int} J_{j}\right.} .
$$

In this paper, we consider a class of one-dimensional transformations that are piecewise linear Markov transformations. Consequently, the transfer operator has the following matrix representation. Let $\mathscr{C}$ be the class of all functions that are piecewise constant on the partition $\mathscr{P}_{I}^{\prime}$. Thus,

$$
g \in \mathscr{C} \quad \text { iff } g=\sum_{j=1}^{m} \pi_{j} \chi_{J_{j}}
$$

for some constants $\pi_{1}, \ldots, \pi_{m}$. We remark that $g$ will also be represented by the column vector $\pi_{g}=\left(\pi_{1}, \ldots, \pi_{m}\right)^{T}$. Using formula (2.34) and considering $g \in \mathscr{C}$ with $g=\chi_{J_{k}}$ for some $1 \leq k \leq m$, the transfer operator $L_{\phi}$ has the following matrix characterization:

$$
L_{\phi} g=Q_{\beta} \pi_{g}
$$

for the weighted dynamical system associated to $\left(\Sigma_{A}, \sigma\right)$. If $D_{\beta}$ is the diagonal matrix defined by

$$
D_{\beta}:=\left(\exp \varphi_{1}^{-\beta}, \ldots, \exp \varphi_{m}^{-\beta}\right)
$$

and $A$ is the transition matrix, then the matrix $Q_{\beta}$ is the $m \times m$ weighted transition matrix defined by

$$
Q_{\beta}:=A D_{\beta}=\left[q_{i j}\right]
$$

The entries of this matrix are

$$
q_{i j}:=\frac{a_{i j}}{\left|F_{j}^{\prime}\right|^{\beta}},
$$

where the derivative $F_{j}^{\prime}$ is evaluated on the interval $J_{j}$ of the partition $\mathscr{P}_{I}^{\prime}$. We refer to $[3,13]$ and the references therein to other important spectral properties of the transfer operator, and [4] for this operator with respect to the cookie-cutter system. In [8], we use the matrix $Q_{\beta}$ with $\beta=1$ to compute the escape rate and the conditional invariant measure which generates the unique invariant probability measure.

There is an isomorphism between $\left(\Sigma_{A}, \sigma\right)$ and $\left(\mathscr{P}_{I}^{\prime}, F\right)$ (see [15]). If $w=\left(i_{0} i_{1} \cdots\right)$ and $w^{\prime}=\left(i_{0}^{\prime} i_{1}^{\prime} \cdots\right)$ are two points of $\Sigma_{A}$, then we consider the Markov partition defined by $\mathscr{P}_{I}^{\prime}:=\Sigma_{A} / \sim$, where $w \sim w^{\prime}$ if and only if $i_{0}=i_{0}^{\prime}$. Using this isomorphism, we consider the trace of the transfer operator defined by

$$
\operatorname{Tr} L_{\phi}:=\sum_{x \in \operatorname{Fix}(F)} \exp \phi(x)
$$


where $\operatorname{Fix}(F)$ denotes the set of fixed points of $F$. We consider the pressure function of $\phi(x)=\log \left|F^{\prime}(x)\right|^{-\beta}$ as $\beta$ varies, $P(\beta)$, defined by

$$
\begin{aligned}
P(\beta) & :=\lim _{k \rightarrow \infty} \frac{1}{k} \log \sum_{x \in \operatorname{Fix}\left(F^{k}\right)}\left|\left(F^{k}\right)^{\prime}(x)\right|^{-\beta} \\
& =\lim _{k \rightarrow \infty} \frac{1}{k} \log \left(\operatorname{Tr} Q_{\beta}^{k}\right) \\
& =\log \left(\lambda_{\beta}\right)
\end{aligned}
$$

where $\operatorname{Fix}\left(F^{k}\right)$ denotes the set of fixed points of $F^{k}$ (see $\left.[4,12,15]\right)$. Thus, $\exp P(\beta)$ is the largest eigenvalue $\lambda_{\beta}$ of the transfer operator $L_{\phi}$, which is equal to the spectral radius of the matrix $Q_{\beta}$ (see [13]). Nevertheless, it is known that the pressure can be characterized by the variational principle

$$
P(\beta)=h_{\mu_{\beta}}(F)-\beta \chi_{\mu_{\beta}}(F)
$$

as the supremum over all invariant probability measures on $E$. In this case, the supremum is attained by the weighted Markov measure $\mu_{\beta}$, that is, the measure $\mu_{\beta}$ is the unique measure that maximizes this expression. See [8] for the definition of $\mu_{\beta}$, the weighted metric entropy $h_{\mu_{\beta}}(F)$, and the weighted Lyapunov exponent $\chi_{\mu_{\beta}}(F)$ with respect to this measure.

We remark that the weighted zeta function for a weighted subshift of finite type is given by

$$
\zeta(t, \beta)=\frac{1}{\operatorname{det}\left(I-t Q_{\beta}\right)}
$$

For more discussions about the zeta function for a subshift of finite type without weights, see [2], and for another approach with weights, see [1, 7].

3. Complexes and homology with weights. As above described, on the set $\left\{b_{1}, \ldots\right.$, $\left.b_{m+1}\right\}$, there exist $2 n-2$ points

$$
\left\{x^{(1)}, \ldots, x^{(2 h-2)}, x^{(2 h+1)}, \ldots, x^{(2 n)}\right\} .
$$

Concerning the set of points $\left\{a_{1}, \ldots, a_{n+1}\right\}$, we consider the respective $2 n-2$ lateral points as in Section 2. Let $p$ be the number of points $b_{i}$ outside of (3.1). We denote this set by $\mathscr{G}$. Set $2 n+p-2=q$ and

$$
\begin{aligned}
\left\{a_{1}^{+},\right. & \left.a_{2}^{-}, a_{2}^{+}, \ldots, a_{h}^{-}, a_{h}^{+}, a_{h+1}^{-}, a_{h+1}^{+}, \ldots, a_{n+1}^{-}\right\} \cup \mathscr{G} \\
& =\left\{o\left(x^{(j)}\right): j=1, \ldots, 2 h-2,2(h+1)-1, \ldots, 2 n\right\} \cup\left\{x^{(2 h-1)}, x^{(2(h+1)-2)}\right\} \\
& =\left\{x^{(1)}, x_{1}^{(1)}, \ldots, x_{k_{1}}^{(1)}, \ldots, x^{(2 n)}, x_{1}^{(2 n)}, \ldots, x_{k_{2 n}}^{(2 n)}\right\} \cup\left\{x^{(2 h-1)}, x^{(2(h+1)-2)}\right\} \\
& =\left\{z^{(1)}, z^{(2)}, \ldots, z^{(q-2)}, x^{(2 h-1)}, x^{(2(h+1)-2)}\right\} \\
& =\left\{z^{(1)}, z^{(2)}, \ldots, z^{(q)}\right\} .
\end{aligned}
$$


We denote by $\left\{y^{(1)}, y^{(2)}, \ldots, y^{(q)}\right\}$ the above set of points now ordered on the interval $I$. On the set $\left\{y^{(1)}, y^{(2)}, \ldots, y^{(q)}\right\}$, there exist pairs of consecutive points $y^{(k)}, y^{(k+1)}$, with $2 \leq k \leq q-2$, correspondent to some $a_{i}^{ \pm}$with $i=2, \ldots, n$. According to the above conditions, we define a permutation $\rho$ by

$$
\left(z^{(1)}, z^{(2)}, \ldots, z^{(q)}\right) \longrightarrow\left(z^{\rho(1)}, z^{\rho(2)}, \ldots, z^{\rho(q)}\right)=\left(y^{(1)}, y^{(2)}, \ldots, y^{(q)}\right)
$$

Let $C_{0}$ be the vector space of 0 -chains spanned by the points $y^{(1)}, \ldots, y^{(q)}$ and $C_{1}$ the vector space of 1 -chains spanned by the intervals of the partition $\mathscr{P}_{I}^{\prime}$. The border map $\partial: C_{1} \rightarrow C_{0}$ is defined by

$$
\partial\left(J_{j}\right):=y^{(i+1)}-y^{(i)}
$$

with $1 \leq j \leq m$ and $1 \leq i \leq q-1$. We designate by $B=\left[b_{i j}\right]$ the $q \times m$ incidence matrix of the graph defined by $\left(C_{0}, C_{1}, \partial\right)$, that is, if $y^{(i)}$ is the lower endpoint and $y^{(i+1)}$ is the upper endpoint of the interval $J_{j}$, then $b_{i j}:=-1$ and $b_{i+1, j}:=1$, and the remaining entries are zero.

Let $\eta: C_{0} \rightarrow C_{0}$ be the map that describes the transition between the points $y^{(1)}$, $y^{(2)}, \ldots, y^{(q)}$ and checks the existence of turning points and discontinuity points between $y^{(j)}$ and $F\left(y^{(j)}\right)$, with $1 \leq j \leq q$. This map is represented by the following weighted matrix $V_{\beta}$.

Definition 3.1. The $(q \times q)$-weighted matrix $V_{\beta}=\left[v_{i j}\right]$, associated to the map $F$, is defined by

$$
\begin{gathered}
v_{i j}:=\varepsilon\left(y^{(j)}\right)\left|F^{\prime}\left(y^{(j)}\right)\right|^{-\beta} \quad \text { if } F\left(y^{(j)}\right)=y^{(i)} \text {, where } \varepsilon\left(y^{(j)}\right)=\operatorname{sign}\left(F^{\prime}\left(y^{(j)}\right)\right), \\
v_{k j}:=v_{i j}, \quad v_{k+1, j}:=-v_{i j} \quad \text { if } y^{(i)}>y^{(j)}, j \leq k<i, \\
v_{k-1, j}:=-v_{i j}, \quad v_{k, j}:=v_{i j} \quad \text { if } y^{(i)}<y^{(j)}, i<k \leq j,
\end{gathered}
$$

where the pairs of consecutive points $y^{(k)}, y^{(k+1)}$, with $j \leq k<i$ (resp., $y^{(k-1)}, y^{(k)}$, with $i<k \leq j$ ), are associated to the turning points and discontinuity points of $F$ with $y^{(j)} \leq y^{(k)}, y^{(k+1)} \leq y^{(i)}$ (resp., $\left.y^{(i)} \leq y^{(k-1)}, y^{(k)} \leq y^{(j)}\right)$. All the remaining entries in $V_{\beta}$ are zero.

We consider that the points $y^{(i)}$, with $1 \leq i \leq q$, are represented by $y^{(i)}=(0, \ldots, 0,1$, $0, \ldots, 0)^{T}$, where 1 is in the $i$ th-position. The above weighted matrices are related by the next result.

LEMMA 3.2. The next diagram is commutative

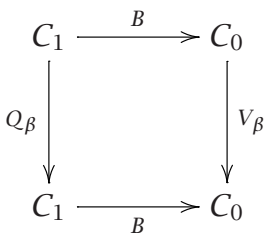


Proof. Let the intervals $J_{j}$, with $1 \leq j \leq m$, be represented by the column vector $J_{j}=\left(0, \ldots, 0, \chi_{J_{j}}, 0, \ldots, 0\right)^{T}$, where $\chi_{J_{j}}$ is in the $j$ th-position, that is, as a function in $\mathscr{C}$. Then, one has

$$
\begin{aligned}
\left(B Q_{\beta}\right)\left(J_{j}\right) & =\left(B A D_{\beta}\right)\left(J_{j}\right) \\
& =(B A)\left(e^{\varphi_{j}^{-\beta}} J_{j}\right) \\
& =B\left(\sum_{a_{k j} \neq 0}\left|F^{\prime}\left(J_{j}\right)\right|^{-\beta} J_{k}\right) \\
& =\sum_{a_{k j} \neq 0}\left|F^{\prime}\left(J_{j}\right)\right|^{-\beta} B\left(J_{k}\right) \\
& =\sum_{u_{l j} \neq 0}\left|F^{\prime}\left(J_{j}\right)\right|^{-\beta}\left(y^{(l+1)}-y^{(l)}\right),
\end{aligned}
$$

where $u_{l j}$ are the nonzero elements of the $j$ th column of the matrix $B A$. The above equalities make a description of the transition of the interval $J_{j}$ by the border of the intervals $J_{k}$ such that $F$ (int $\left.J_{j}\right) \supseteq \operatorname{int} J_{k}$, weighted by $\left|F^{\prime}\left(J_{j}\right)\right|^{-\beta}$.

On the other hand, we have

$$
V_{\beta} B\left(J_{j}\right)=V_{\beta}\left(y^{(i+1)}-y^{(i)}\right)=V_{\beta}\left(y^{(i+1)}\right)-V_{\beta}\left(y^{(i)}\right) .
$$

Consider that $y^{(i)}$ is a point associated to a turning point or to a discontinuity point of $F$ and $F\left(y^{(i)}\right)=y^{(s)}$, with $s<i$. Consequently,

$$
v_{s i}=\varepsilon\left(y^{(i)}\right)\left|F^{\prime}\left(y^{(i)}\right)\right|^{-\beta}, \quad v_{i-1, i}=-v_{s i}, \quad v_{i i}=v_{s i}
$$

If there exist $z_{1}$ turning points or discontinuity points between $y^{(s)}$ and $y^{(i-1)}$, then we have pairs of consecutive points $y^{\left(k_{l}-1\right)}, y^{\left(k_{l}\right)}$, with $s<k_{l}-1, k_{l}<i-1$ and $1 \leq l \leq z_{1}$ such that

$$
v_{k_{l}-1, i}=-v_{s i}, \quad v_{k_{l} i}=v_{s i}
$$

Suppose that $y^{(i+1)} \in \operatorname{int} I_{p}$, with $1 \leq p \leq n$, that is, $y^{(i+1)} \in \mathscr{G}$ and $F\left(y^{(i+1)}\right)=y^{(r)}$, with $r>i+1$. In this case, we have

$$
v_{i+1, i+1}=0, \quad v_{r, i+1}=\varepsilon\left(y^{(i+1)}\right)\left|F^{\prime}\left(y^{(i+1)}\right)\right|^{-\beta} .
$$

Similarly, if there exist $z_{2}$ turning points or discontinuity points between $y^{(i+1)}$ and $y^{(r)}$, then we have pairs of points $y^{\left(k_{w}\right)}, y^{\left(k_{w}+1\right)}$, with $i+1<k_{w}, k_{w}+1<r$ and $1 \leq w \leq z_{2}$ such that

$$
v_{k_{w} i}=v_{r, i+1}, \quad v_{k_{w}+1, i}=-v_{r, i+1} .
$$

As the weight is constant on each interval $J_{j}$, we get

$$
V_{\beta}\left(y^{(i+1)}\right)-V_{\beta}\left(y^{(i)}\right)=\left|F^{\prime}\left(J_{j}\right)\right|^{-\beta} \sum_{l=s}^{r-1}\left(y^{(l+1)}-y^{(l)}\right),
$$


where the pairs of points $y^{(l)}, y^{(l+1)}$ lie in the set

$$
\left\{y^{(s)}, y^{(s+1)}, \ldots, y^{\left(k_{l}\right)}, y^{\left(k_{l}+1\right)}, \ldots, y^{(i-1)}, y^{(i)}, \ldots, y^{\left(k_{w}\right)}, y^{\left(k_{w}+1\right)}, \ldots, y^{(r-1)}, y^{(r)}\right\}
$$

and describe the border of the intervals $J_{k}$ such that $F$ (int $\left.J_{j}\right) \supseteq \operatorname{int} J_{k}$. From this, it follows that

$$
\left|F^{\prime}\left(J_{j}\right)\right|^{-\beta} \sum_{l=s}^{r-1}\left(y^{(l+1)}-y^{(l)}\right)=\sum_{a_{k j} \neq 0}\left|F^{\prime}\left(J_{j}\right)\right|^{-\beta} B\left(J_{k}\right) .
$$

The proof of the remaining cases is similar according to the behavior of $F$ and the above definition.

Let $H_{0}:=C_{0} / B_{0}$, where $B_{0}=\partial\left(C_{1}\right)$ is a subspace of $C_{0}$. Note that two consecutive laps without a discontinuity point between them are considered as two connected components. The map $\zeta: C_{0} \rightarrow H_{0}$ associates to each point $y^{(i)}$, with $1 \leq i \leq q$, the respective interval $I_{j}$, with $1 \leq j \leq n$. This map is represented by the $q \times n$ matrix $U=\left[u_{i j}\right]$, where $u_{i j}:=1$ if the point $y^{(i)}$ lies in $I_{j}$ and all the remaining entries of the matrix are zero.

LEMMA 3.3. If $y^{\left(i_{1}\right)}$ and $y^{\left(i_{2}\right)}$ are two points on the interval $I_{j}$ with $1 \leq j \leq n$, then

$$
U V_{\beta}\left(y^{\left(i_{1}\right)}\right)^{T}=U V_{\beta}\left(y^{\left(i_{2}\right)}\right)^{T}
$$

Proof. Let $y^{\left(i_{1}\right)}$ and $y^{\left(i_{2}\right)}$ be two points on $I_{j}$. Consider that

$$
B\left(J_{k}\right)=y^{\left(i_{2}\right)}-y^{\left(i_{1}\right)}, \quad \operatorname{int} J_{k} \subseteq \operatorname{int} I_{j}, \quad F\left(y^{\left(i_{1}\right)}\right) \in I_{j^{\prime}}, \quad F\left(y^{\left(i_{2}\right)}\right) \in I_{j^{\prime \prime}},
$$

with $1 \leq k \leq m$ and $j^{\prime}<j<j^{\prime \prime}$. If the points $y^{\left(i_{1}\right)}$ and $y^{\left(i_{2}\right)}$ have the same behavior under $F$ as $y^{(i)}$ and $y^{(i+1)}$ in the proof of the above proposition, respectively, then

$$
\begin{aligned}
U V_{\beta}\left(y^{\left(i_{1}\right)}\right)^{T} & =U\left(0, \ldots, 0, v_{s i_{1}}, \ldots, v_{k_{l}-1, i_{1}}, v_{k_{l} i_{1}}, \ldots, v_{i_{1}-1, i_{1}}, v_{i_{1} i_{1}}, 0, \ldots, 0\right)^{T} \\
& =U \varepsilon\left(y^{\left(i_{1}\right)}\right)\left|F^{\prime}\left(y^{\left(i_{1}\right)}\right)\right|^{-\beta}(0, \ldots, 0,1, \ldots,-1,1, \ldots,-1,1,0, \ldots, 0)^{T} .
\end{aligned}
$$

Note that there exist $z_{1}$ pairs of consecutive points $y^{\left(k_{l}-1\right)}, y^{\left(k_{l}\right)}$ between $y^{(s)}$ and $y^{\left(i_{1}-1\right)}$, with $1 \leq l \leq z_{1}$. These points define the border of the $p$ intervals $I_{j t}$, with $j^{\prime}<j_{t}<j$ and $1 \leq t \leq p$. This implies that

$$
\begin{gathered}
B\left(I_{j^{\prime}}\right)=y^{\left(k_{1}-1\right)}-y^{(s)}, \\
B\left(I_{j_{1}}\right)=y^{\left(k_{2}-1\right)}-y^{\left(k_{1}\right)}, \\
\vdots \\
B\left(I_{j}\right)=y^{\left(i_{1}\right)}-y^{\left(i_{1}-1\right)} .
\end{gathered}
$$


Thus, by definition of $U$, we get

$$
U V_{\beta}\left(y^{\left(i_{1}\right)}\right)^{T}=\left(0, \ldots, 0, \varepsilon\left(y^{\left(i_{1}\right)}\right)\left|F^{\prime}\left(y^{\left(i_{1}\right)}\right)\right|^{-\beta}, 0, \ldots, 0\right)^{T},
$$

where $\varepsilon\left(y^{\left(i_{1}\right)}\right)\left|F^{\prime}\left(y^{\left(i_{1}\right)}\right)\right|^{-\beta}$ is in the $j$ th-position.

On the other hand, considering the point $y^{\left(i_{2}\right)}$, we can write

$$
\begin{aligned}
U V_{\beta}\left(y^{\left(i_{2}\right)}\right)^{T} & =U\left(0, \ldots, 0, v_{k_{w} i_{2}}, v_{k_{w}+1, i_{2}}, \ldots, v_{r i_{2}}, 0, \ldots, 0\right)^{T} \\
& =U \varepsilon\left(y^{\left(i_{2}\right)}\right)\left|F^{\prime}\left(y^{\left(i_{2}\right)}\right)\right|^{-\beta}(0, \ldots, 0,1,-1, \ldots, 1,0, \ldots, 0)^{T} .
\end{aligned}
$$

Note that there exist $z_{2}$ pairs of consecutive points $y^{\left(k_{w}\right)}, y^{\left(k_{w}+1\right)}$ between $y^{\left(i_{2}\right)}$ and $y^{(r)}$, with $1 \leq w \leq z_{2}$. Similarly and by definition of $U$, we verify that

$$
U V_{\beta}\left(y^{\left(i_{2}\right)}\right)^{T}=\left(0, \ldots, 0, \varepsilon\left(y^{\left(i_{2}\right)}\right)\left|F^{\prime}\left(y^{\left(i_{2}\right)}\right)\right|^{-\beta}, 0, \ldots, 0\right)^{T},
$$

where $\varepsilon\left(y^{\left(i_{2}\right)}\right)\left|F^{\prime}\left(y^{\left(i_{2}\right)}\right)\right|^{-\beta}$ is in the $j$ th-position. As the weight is constant on each interval $I_{j}$, the desired result follows. Nevertheless, according to the behavior of $F$, several different cases may occur. The proof of the remaining cases is very similar to the previous one.

The above lemma suggests the next definition and result. Associated to each matrix $V_{\beta}$, we have only one map $\xi: H_{0} \rightarrow H_{0}$ which reflects the monotonicity of $F$. The map $\xi$ is represented by the $(n \times n)$-weighted diagonal matrix $K_{\beta}=\left[k_{i j}\right]$, where

$$
k_{i i}:=\varepsilon\left(I_{i}\right)\left|F^{\prime}\left(I_{i}\right)\right|^{-\beta},
$$

with $\varepsilon\left(I_{i}\right)=\operatorname{sign}\left(F^{\prime}(x)\right), x \in I_{i}$.

LEMMA 3.4. The next diagram is commutative

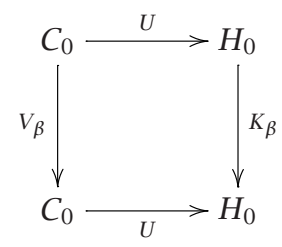

The main results can now be stated. The next theorems establish the relation between the weighted transition matrix, the weighted matrix $V_{\beta}$, and the weighted kneading determinant.

THEOREM 3.5. Under the conditions of the previous lemmas, the following relation holds between the characteristic polynomials of the matrices $Q_{\beta}, V_{\beta}$, and $K_{\beta}$ :

$$
P_{V_{\beta}}(t)=\operatorname{det}\left(I-t V_{\beta}\right)=P_{Q_{\beta}}(t) P_{K_{\beta}}(t)
$$

Proof. The statement is a consequence of the above lemmas and according to some homological algebra results (see [5]). 
We define a permutation matrix $\Pi$ associated to the permutation $\rho$ above defined that maps the system of vectors $\left(z^{(1)}, z^{(2)}, \ldots, z^{(q)}\right)$ into the system of vectors $\left(y^{(1)}, y^{(2)}, \ldots\right.$, $\left.y^{(q)}\right)$. Using the weighted matrix $V_{\beta}$ and the permutation matrix $\Pi$, we define a new weighted matrix $\Theta_{\beta}$ through the next equality:

$$
\Theta_{\beta}:=\Pi V_{\beta} \Pi^{T}
$$

The matrix $\Pi$ is invertible; consequently, we have $P_{\Theta_{\beta}}(t)=P_{V_{\beta}}(t)$.

THEOREM 3.6. If the kneading data associated to an expanding discontinuous map with a hole F corresponds to periodic, eventually periodic orbits, or to orbits that lie in the hole, then the weighted kneading determinant is given by

$$
D(t, \beta)=\frac{P_{Q_{\beta}}(t)}{R(t)},
$$

where $R(t)$ is a product of weighted cyclotomic polynomials correspondent to those periodic or eventually periodic orbits.

It is obvious that this statement strongly depends on the number of laps and the kneading data associated to $F$. For this reason, the analysis of the general situation is difficult. We will prove the statement for a map $F=\left(F_{1}, F_{2}\right)$. The general case follows in a similar way.

Proof. Consider $F=\left(F_{1}, F_{2}\right), \mathscr{P}_{I}=\left\{I_{1}, I_{2}, I_{3}\right\}$, where $I_{2}$ is the hole, $F_{1}\left(a_{2}\right)=1$, $F_{2}\left(a_{3}\right)=0$, and $\mathscr{A}=\{L, H, R\}$. The orbits of the points $a_{1}^{+}$and $a_{4}^{-}$can be periodic, eventually periodic, or lie in the hole. We consider that the kneading data associated to this map is given by

$$
\left(o\left(x^{(2)}\right), o\left(x^{(5)}\right)\right)=\left(\left(L S_{1}^{(2)} \cdots S_{p-1}^{(2)}\right)^{\infty},\left(M S_{1}^{(5)} \cdots S_{q-1}^{(5)}\right)^{\infty}\right),
$$

where $p$ and $q$ are the periods of the orbits. The weighted kneading increments are

$$
v_{a_{2}}(t, \beta)=\theta_{a_{2}^{-}}(t, \beta), \quad v_{a_{3}}(t, \beta)=\theta_{a_{3}^{+}}(t, \beta),
$$

where

$$
\begin{aligned}
& \theta_{a_{2}^{-}}(t, \beta)=\frac{L+\sum_{i=1}^{p-1} \tau_{i}\left(x^{(2)}\right) t^{i} S_{i}^{(2)}}{1-\tau_{p}\left(x^{(2)}\right) t^{p}}, \\
& \theta_{a_{3}^{+}}(t, \beta)=\frac{R+\sum_{i=1}^{q-1} \tau_{i}\left(x^{(5)}\right) t^{i} S_{i}^{(5)}}{1-\tau_{q}\left(x^{(5)}\right) t^{q}} .
\end{aligned}
$$

If we write

$$
L_{p}=\sum_{\substack{i=1 \\ S_{i}^{(2)}=L}}^{p-1} \tau_{i}\left(x^{(2)}\right) t^{i}, \quad R_{p}=\sum_{\substack{i=1 \\ S_{i}^{(2)}=R}}^{p-1} \tau_{i}\left(x^{(2)}\right) t^{i}
$$


and analogously for $L_{q}$ and $R_{q}$, then we have

$$
\begin{aligned}
& v_{a_{2}}(t, \beta)=\frac{1+L_{p}}{1-\tau_{p}\left(x^{(2)}\right) t^{p}} L+\frac{R_{p}}{1-\tau_{p}\left(x^{(2)}\right) t^{p}} R, \\
& v_{a_{3}}(t, \beta)=\frac{L_{q}}{1-\tau_{q}\left(x^{(5)}\right) t^{q}} L+\frac{1+R_{q}}{1-\tau_{q}\left(x^{(5)}\right) t^{q}} R .
\end{aligned}
$$

The weighted kneading determinant $D(t, \beta)$ for these kneading data is

$$
D(t, \beta)=\frac{1}{\left(1-\tau_{p}\left(x^{(2)}\right) t^{p}\right)\left(1-\tau_{q}\left(x^{(5)}\right) t^{q}\right)}\left|\begin{array}{cc}
1+L_{p} & R_{p} \\
L_{q} & 1+R_{q}
\end{array}\right| .
$$

Let $\operatorname{det} \bar{\Theta}_{\beta}=\operatorname{det}\left(I-t \Theta_{\beta}\right)$ be the characteristic polynomial of the matrix $\Theta_{\beta}$, where $I$ is the identity matrix. Thus, we have

$$
\bar{\Theta}_{\beta}=\left[\begin{array}{cccccccccc}
1-\mu_{2,0} t & -\mu_{2,1} t & \cdots & -\mu_{2, p-2} t & -\mu_{2, p-1} t-\theta_{2, p-1} t & -\mu_{5,0} t & -\mu_{5,1} t & \cdots & -\mu_{5, q-2} t & -\mu_{5, q-1} t \\
-\theta_{2,0} t & 1 & \cdots & 0 & 0 & 0 & 0 & \cdots & 0 & 0 \\
\vdots & \vdots & \ddots & \vdots & \vdots & \vdots & \vdots & \ddots & \vdots & \vdots \\
0 & 0 & \cdots & 1 & 0 & 0 & 0 & \cdots & 0 & 0 \\
0 & 0 & \cdots & -\theta_{2, p-2} t & 1 & 0 & 0 & \cdots & 0 & 0 \\
-\delta_{2,0} t & -\delta_{2,1} t & \cdots & -\delta_{2, p-2} t & -\delta_{2, p-1} t & 1-\delta_{5,0} t & -\delta_{5,1} t & \cdots & -\delta_{5, q-2} t & -\delta_{5, q-1} t-\theta_{5, q-1} t \\
0 & 0 & \cdots & 0 & 0 & -\theta_{5,0} t & 1 & \cdots & 0 & 0 \\
\vdots & \vdots & \ddots & \vdots & \vdots & \vdots & \vdots & \ddots & \vdots & \vdots \\
0 & 0 & \cdots & 0 & 0 & 0 & 0 & \cdots & 1 & 0 \\
0 & 0 & \cdots & 0 & 0 & 0 & 0 & \cdots & -\theta_{5, q-2} t & 1
\end{array}\right],
$$

where

$$
\begin{aligned}
& \theta_{2, k_{1}}=\varepsilon\left(x_{k_{1}}^{(2)}\right)\left|F^{\prime}\left(x_{k_{1}}^{(2)}\right)\right|^{-\beta}, \\
& \mu_{2, k_{1}} \in\left\{0, \pm \varepsilon\left(x_{k_{1}}^{(2)}\right)\left|F^{\prime}\left(x_{k_{1}}^{(2)}\right)\right|^{-\beta}\right\}, \quad \text { with } 0 \leq k_{1} \leq p-1, \\
& \theta_{5, k_{2}}=\varepsilon\left(x_{k_{2}}^{(5)}\right)\left|F^{\prime}\left(x_{k_{2}}^{(5)}\right)\right|^{-\beta}, \\
& \mu_{5, k_{2}} \in\left\{0, \pm \varepsilon\left(x_{k_{2}}^{(5)}\right)\left|F^{\prime}\left(x_{k_{2}}^{(5)}\right)\right|^{-\beta}\right\}, \quad \text { with } 0 \leq k_{2} \leq q-1,
\end{aligned}
$$

and similarly for $\delta_{2, k_{1}}$ and $\delta_{5, k_{2}}$. Using matrix elementary operations for the matrix $I-t \Theta_{\beta}$, we have the following equivalent matrix:

$$
\left[\begin{array}{cc}
1-\sum_{k=0}^{p-1} \mu_{2, k} \boldsymbol{T}_{k}\left(x_{0}^{(2)}\right) t^{k+1}-\tau_{p}\left(x_{0}^{(2)}\right) t^{p} & -\sum_{k=0}^{q-1} \mu_{5, k} \boldsymbol{T}_{k}\left(x_{0}^{(5)}\right) t^{k+1} \\
-\sum_{k=0}^{p-1} \delta_{2, k} \tau_{k}\left(x_{0}^{(2)}\right) t^{k+1} & 1-\sum_{k=0}^{q-1} \delta_{5, k} \tau_{k}\left(x_{0}^{(5)}\right) t^{k+1}-\tau_{q}\left(x_{0}^{(5)}\right) t^{q}
\end{array}\right]
$$


Now we will compare the elements of the above matrix to the elements of the weighted kneading matrix $N(t, \beta)$. Note that

$$
\begin{aligned}
1-\sum_{k=0}^{p-1} \mu_{2, k} \boldsymbol{\tau}_{k}\left(x_{0}^{(2)}\right) t^{k+1}-\boldsymbol{\tau}_{p}\left(x_{0}^{(2)}\right) t^{p} \\
=\left(1-\mu_{2,0} t\right)+\sum_{k=1}^{p-1} \tau_{k}\left(x_{0}^{(2)}\right) t^{k}\left(-\mu_{2, k} t\right) \\
\quad+\tau_{p-1}\left(x_{0}^{(2)}\right) t^{p-1}\left(-\varepsilon\left(x_{p-1}^{(2)}\right)\left|F^{\prime}\left(x_{p-1}^{(2)}\right)\right|^{-\beta} t\right) .
\end{aligned}
$$

On the other hand,

$$
\begin{aligned}
\left(1-\varepsilon\left(I_{1}\right)\left|F^{\prime}\left(I_{1}\right)\right|^{-\beta} t\right)\left(1+L_{p}\right) & \left(1-\varepsilon\left(I_{1}\right)\left|F^{\prime}\left(I_{1}\right)\right|^{-\beta} t\right)+\sum_{\substack{k=1 \\
S_{k}^{(2)}=L}}^{p-1} \tau_{k}\left(x_{0}^{(2)}\right) t^{k} \\
= & +\sum_{\substack{k=1 \\
S_{k}^{(2)}=L}}^{p-1} \tau_{k}\left(x_{0}^{(2)}\right) t^{k}\left(-\varepsilon\left(I_{1}\right)\left|F^{\prime}\left(I_{1}\right)\right|^{-\beta} t\right) .
\end{aligned}
$$

If $\mu_{2, k} \neq 0$, with $1 \leq k \leq p-1$, then $\tau_{k+1}\left(x_{0}^{(2)}\right) \neq 0$ on $L_{p}$, that is,

$$
\tau_{k}\left(x_{0}^{(2)}\right) t^{k}\left( \pm \varepsilon\left(x_{k}^{(2)}\right)\left|F^{\prime}\left(x_{k}^{(2)}\right)\right|^{-\beta} t\right)= \pm \tau_{k+1}\left(x_{0}^{(2)}\right) t^{k+1} \text {. }
$$

In particular, if $\mu_{2, p-1} \neq 0$, then $x_{p-1}^{(2)}$ is associated to the symbol $R$, that is,

$$
\mu_{2, p-1}=-\varepsilon\left(x_{p-1}^{(2)}\right)\left|F^{\prime}\left(x_{p-1}^{(2)}\right)\right|^{-\beta} .
$$

Consequently, in (3.37), we have

$$
\tau_{p-1}\left(x_{0}^{(2)}\right) t^{p-1} \varepsilon\left(x_{p-1}^{(2)}\right)\left|F^{\prime}\left(x_{p-1}^{(2)}\right)\right|^{-\beta} t=\tau_{p}\left(x_{0}^{(2)}\right) t^{p} .
$$

Hence, in (3.38), the fact that the orbit is periodic implies that we return to the symbol $L$. Thus, we have

$$
1-\mu_{2,0} t=1-\varepsilon\left(I_{1}\right)\left|F^{\prime}\left(I_{1}\right)\right|^{-\beta} t .
$$

Let $R(t)$ be the product of cyclotomic polynomials and $P_{K_{\beta}}(t)$ the characteristic polynomial of the matrix $K_{\beta}$ associated to $F$. Set

$$
D^{*}(t, \beta)=\left|\begin{array}{cc}
\left(1-\varepsilon\left(I_{1}\right)\left|F^{\prime}\left(I_{1}\right)\right|^{-\beta} t\right)\left(1+L_{p}\right) & \left(1-\varepsilon\left(I_{2}\right)\left|F^{\prime}\left(I_{2}\right)\right|^{-\beta} t\right) R_{p} \\
\left(1-\varepsilon\left(I_{1}\right)\left|F^{\prime}\left(I_{1}\right)\right|^{-\beta} t\right) L_{q} & \left(1-\varepsilon\left(I_{2}\right)\left|F^{\prime}\left(I_{2}\right)\right|^{-\beta} t\right)\left(1+R_{q}\right)
\end{array}\right| .
$$


Using the above comparison between the elements of the equivalent matrix to $I-t \Theta_{\beta}$ and the elements of the weighted kneading matrix, we have

$$
D(t, \beta)=\frac{D^{*}(t, \beta)}{R(t) P_{K_{\beta}}(t)}=\frac{\operatorname{det}\left(I-t \Theta_{\beta}^{T}\right)}{R(t) P_{K_{\beta}}(t)}=\frac{P_{V_{\beta}}(t)}{R(t) P_{K_{\beta}}(t)} .
$$

By Theorem 3.5, the desired result follows.

The main result, Theorem 1.1, will allows us to compute explicitly the Hausdorff dimension, the escape rate, and the topological entropy.

Proof of TheOrem 1.1. Considering the transfer operator given in (2.34), we have

$$
\left.\left(L_{\phi_{j}} g\right)(x)=\sum_{j=1}^{m}\left|F_{j}^{\prime}(x)\right|^{-\beta} g\left(f_{j}(x)\right) \chi_{F\left(\operatorname{int} J_{j}\right.}\right)
$$

Let $a_{i j}$ be the entries of the transition matrix $A$. For each $J_{i} \in \mathscr{P}_{I}^{\prime}$, with $1 \leq i \leq m$ and $\beta \in \mathbb{R}$, the eigenvalue equation corresponding to an eigenvalue $\lambda_{\beta}$ is

$$
\sum_{j=1}^{m} \frac{a_{i j}}{\left|F_{j}^{\prime}(x)\right|^{\beta}} v_{j}=\lambda_{\beta} v_{i}
$$

for the operator $L_{\phi}$ characterized by the matrix $Q_{\beta}$. According to [11] and using (2.41), the largest eigenvalue of the transfer operator is $\exp P(\beta)$. Hence, $\exp P(\beta)$ is the spectral radius $\lambda_{\beta}$ of the matrix $Q_{\beta}$.

If $\beta$ is the unique solution of $D(1, \beta)=0$, then by Theorem 3.6 and (2.41), we get $P(\beta)=0$. By $[4,10]$, we can conclude that $\beta=\operatorname{dim}_{H}(E)$.

On the other hand, considering the parameter $\beta=1$, we have that $\lambda_{1}=\exp P(1)$ is the largest eigenvalue of the matrix $Q_{1}$. The second statement follows from [14], where the escape rate $\gamma$ is given by $\gamma=-P(1)$. Thus, the escape rate is $\gamma=\log \left(\lambda_{1}^{-1}\right)$, where $\lambda_{1}^{-1}=t_{1}$ is the least real positive solution of $P_{Q_{1}}(t)=0$.

If $\beta=0$, then the determinant $D(t, 0)$ corresponds to the kneading determinant described in [9], where $t_{0}^{-1}=\lambda_{0}$ is the growth number of $F$, that is, the spectral radius of the transition matrix $A$. Consequently, $\log \left(\lambda_{0}\right)$ is the topological entropy of the map $F$.

REMARK 3.7. The theory presented in this paper with respect to periodic, eventually periodic orbits, or to the orbits that lie in the hole is also valid for aperiodic orbits. In this case, the invariant coordinates associated to the turning points and to the discontinuity points are formal power series. The computation of the topological invariants is done by approximation using periodic, eventually periodic orbits, or the orbits that lie in the hole.

The above results are illustrated in the next example, showing in detail the techniques under discussion. 
EXAMPLE 3.8. Let

$$
F(x)= \begin{cases}\frac{x}{a} & \text { if } x \in\left[0, \frac{1}{8}\right], \\ -\frac{x}{a}+1 & \text { if } x \in\left[\frac{1}{8}, \frac{1}{4}\right], \\ \frac{x}{b}-\frac{3}{4} & \text { if } x \in\left[\frac{1}{4}, \frac{7}{12}\right], \\ \frac{x}{c}-1 & \text { if } x \in\left[\frac{4}{6}, 1\right],\end{cases}
$$

with $a=1 / 4, b=1 / 3$, and $c=1 / 2$. Considering the orbits of the points $a_{2}^{ \pm}, a_{3}^{ \pm}, a_{4}^{-}$, and $a_{5}^{+}$, the kneading data to the map $F$ are

$$
\left(L\left(M_{2} R\right)^{\infty}, M_{1}\left(M_{2} R\right)^{\infty}, M_{1} L^{\infty}, M_{2} L^{\infty}, M_{2} R^{\infty}, R M_{2} M_{2} L^{\infty}\right) .
$$

The weighted invariant coordinates of each point are

$$
\begin{aligned}
& \theta_{a_{2}^{-}}(t, \beta)=L+\frac{a^{\beta} t}{1-(b c)^{\beta} t^{2}} M_{2}+\frac{(a b)^{\beta} t^{2}}{1-(b c)^{\beta} t^{2}} R, \\
& \theta_{a_{2}^{+}}(t, \beta)=M_{1}-\frac{a^{\beta} t}{1-(b c)^{\beta} t^{2}} M_{2}-\frac{(a b)^{\beta} t^{2}}{1-(b c)^{\beta} t^{2}} R, \\
& \theta_{a_{3}^{-}}(t, \beta)=-\frac{a^{\beta} t}{1-a^{\beta} t} L+M_{1}, \quad \theta_{a_{3}^{+}}(t, \beta)=\frac{b^{\beta} t}{1-a^{\beta} t} L+M_{2}, \\
& \theta_{a_{4}^{-}}(t, \beta)=M_{2}+\frac{b^{\beta} t}{1-c^{\beta} t} R, \quad \theta_{a_{5}^{+}}(t, \beta)=\frac{\left(b^{2} c\right)^{\beta} t^{3}}{1-a^{\beta} t} L+\left(c^{\beta} t+(b c)^{\beta} t^{2}\right) M_{2}+R .
\end{aligned}
$$

Consequently, the weighted kneading determinant is

$$
\begin{aligned}
D(t, \beta)= & {\left[\begin{array}{cccc}
-1 & 1 & \frac{-2 a^{\beta} t}{1-(b c)^{\beta} t^{2}} & \frac{-2(a b)^{\beta} t^{2}}{1-(b c)^{\beta} t^{2}} \\
\frac{a^{\beta} t+b^{\beta} t}{1-a^{\beta} t} & -1 & 1 & 0 \\
0 & 0 & 1 & \frac{b^{\beta} t}{1-c^{\beta} t} \\
\frac{\left(b^{2} c\right)^{\beta} t^{3}}{1-a^{\beta} t} & 0 & c^{\beta} t+(b c)^{\beta} t^{2} & 1
\end{array}\right] } \\
= & \frac{1}{\left(1-a^{\beta} t\right)\left(1-c^{\beta} t\right)\left(1-(b c)^{\beta} t^{2}\right)} \\
& \times\left(1-\left(2 a^{\beta}+b^{\beta}+c^{\beta}\right) t+\left(2(a c)^{\beta}-(b c)^{\beta}\right) t^{2}\right. \\
& +\left(4(a b c)^{\beta}+\left(b^{2} c\right)^{\beta}+\left(b c^{2}\right)^{\beta}\right) t^{3} \\
& \left.+\left(2\left(a b^{2} c\right)^{\beta}-2\left(a b c^{2}\right)^{\beta}\right) t^{4}-2\left(a b^{2} c^{2}\right)^{\beta} t^{5}\right) .
\end{aligned}
$$


The orbits of the points $a_{2}^{ \pm}, a_{3}^{ \pm}, a_{4}^{-}$, and $a_{5}^{+}$determine a Markov partition of [0,1], $\mathscr{P}_{I}^{\prime}=\left\{I_{1}, \ldots, I_{8}\right\}$, where $I_{6}$ is the hole. The matrices correspondent to this map are

$$
\begin{aligned}
& B=\left[\begin{array}{cccccccc}
-1 & 0 & 0 & 0 & 0 & 0 & 0 & 0 \\
1 & 0 & 0 & 0 & 0 & 0 & 0 & 0 \\
0 & -1 & 0 & 0 & 0 & 0 & 0 & 0 \\
0 & 1 & 0 & 0 & 0 & 0 & 0 & 0 \\
0 & 0 & -1 & 0 & 0 & 0 & 0 & 0 \\
0 & 0 & 1 & -1 & 0 & 0 & 0 & 0 \\
0 & 0 & 0 & 1 & -1 & 0 & 0 & 0 \\
0 & 0 & 0 & 0 & 1 & 0 & 0 & 0 \\
0 & 0 & 0 & 0 & 0 & -1 & 0 & 0 \\
0 & 0 & 0 & 0 & 0 & 1 & 0 & 0 \\
0 & 0 & 0 & 0 & 0 & 0 & -1 & 0 \\
0 & 0 & 0 & 0 & 0 & 0 & 1 & -1 \\
0 & 0 & 0 & 0 & 0 & 0 & 0 & 1
\end{array}\right], \\
& Q_{\beta}=\left[\begin{array}{cccccccc}
a^{\beta} & a^{\beta} & b^{\beta} & 0 & 0 & 0 & 0 & 0 \\
a^{\beta} & a^{\beta} & b^{\beta} & 0 & 0 & 0 & 0 & 0 \\
a^{\beta} & a^{\beta} & 0 & b^{\beta} & 0 & 0 & 0 & 0 \\
a^{\beta} & a^{\beta} & 0 & b^{\beta} & 0 & 0 & c^{\beta} & 0 \\
0 & 0 & 0 & b^{\beta} & 0 & 0 & 0 & c^{\beta} \\
0 & 0 & 0 & b^{\beta} & 0 & 0 & 0 & c^{\beta} \\
0 & 0 & 0 & b^{\beta} & 0 & 0 & 0 & c^{\beta} \\
0 & 0 & 0 & 0 & b^{\beta} & 0 & 0 & c^{\beta}
\end{array}\right], \\
& V_{\beta}=\left[\begin{array}{ccccccccccccc}
a^{\beta} & 0 & 0 & -a^{\beta} & b^{\beta} & 0 & 0 & 0 & 0 & 0 & 0 & 0 & 0 \\
0 & a^{\beta} & 0 & a^{\beta} & -b^{\beta} & 0 & 0 & 0 & 0 & 0 & 0 & 0 & 0 \\
0 & -a^{\beta} & 0 & -a^{\beta} & b^{\beta} & 0 & 0 & 0 & 0 & 0 & 0 & 0 & 0 \\
0 & a^{\beta} & -a^{\beta} & 0 & -b^{\beta} & 0 & 0 & 0 & 0 & 0 & 0 & 0 & 0 \\
0 & -a^{\beta} & a^{\beta} & 0 & b^{\beta} & b^{\beta} & 0 & 0 & 0 & 0 & 0 & 0 & 0 \\
0 & 0 & 0 & 0 & 0 & 0 & 0 & 0 & 0 & 0 & c^{\beta} & 0 & 0 \\
0 & a^{\beta} & -a^{\beta} & 0 & 0 & 0 & 0 & 0 & 0 & 0 & 0 & c^{\beta} & 0 \\
0 & 0 & 0 & 0 & 0 & 0 & b^{\beta} & b^{\beta} & 0 & 0 & -c^{\beta} & -c^{\beta} & 0 \\
0 & 0 & 0 & 0 & 0 & 0 & -b^{\beta} & -b^{\beta} & 0 & 0 & c^{\beta} & c^{\beta} & 0 \\
0 & 0 & 0 & 0 & 0 & 0 & b^{\beta} & b^{\beta} & 0 & 0 & -c^{\beta} & -c^{\beta} & 0 \\
0 & 0 & 0 & 0 & 0 & 0 & -b^{\beta} & -b^{\beta} & 0 & 0 & c^{\beta} & c^{\beta} & 0 \\
0 & 0 & 0 & 0 & 0 & 0 & b^{\beta} & 0 & 0 & 0 & 0 & 0 & 0 \\
0 & 0 & 0 & 0 & 0 & 0 & 0 & b^{\beta} & 0 & 0 & 0 & 0 & c^{\beta}
\end{array}\right], \\
& U=\left[\begin{array}{lllllllllllll}
1 & 1 & 0 & 0 & 0 & 0 & 0 & 0 & 0 & 0 & 0 & 0 & 0 \\
0 & 0 & 1 & 1 & 0 & 0 & 0 & 0 & 0 & 0 & 0 & 0 & 0 \\
0 & 0 & 0 & 0 & 1 & 1 & 1 & 1 & 0 & 0 & 0 & 0 & 0 \\
0 & 0 & 0 & 0 & 0 & 0 & 0 & 0 & 0 & 0 & 1 & 1 & 1
\end{array}\right], \quad K_{\beta}=\left[\begin{array}{cccc}
a^{\beta} & 0 & 0 & 0 \\
0 & -a^{\beta} & 0 & 0 \\
0 & 0 & b^{\beta} & 0 \\
0 & 0 & 0 & c^{\beta}
\end{array}\right] \text {, } \\
& \Pi=\left[\begin{array}{ccccccccccccc}
1 & 2 & 3 & 4 & 5 & 6 & 7 & 8 & 9 & 10 & 11 & 12 & 13 \\
6 & 1 & 4 & 5 & 7 & 11 & 2 & 8 & 12 & 13 & 10 & 3 & 9
\end{array}\right],
\end{aligned}
$$




$$
\Theta_{\beta}=\left[\begin{array}{ccccccccccccc}
a^{\beta} & 0 & 0 & 0 & a^{\beta} & 0 & -b^{\beta} & 0 & 0 & 0 & 0 & 0 & 0 \\
a^{\beta} & 0 & c^{\beta} & -a^{\beta} & 0 & 0 & 0 & 0 & 0 & 0 & 0 & 0 & 0 \\
0 & b^{\beta} & 0 & 0 & 0 & 0 & 0 & 0 & 0 & 0 & 0 & 0 & 0 \\
-a^{\beta} & 0 & 0 & 0 & -a^{\beta} & 0 & b^{\beta} & 0 & 0 & 0 & 0 & 0 & 0 \\
a^{\beta} & 0 & 0 & -a^{\beta} & 0 & 0 & -b^{\beta} & 0 & 0 & 0 & 0 & 0 & 0 \\
0 & 0 & 0 & 0 & -a^{\beta} & a^{\beta} & b^{\beta} & 0 & 0 & 0 & 0 & 0 & 0 \\
-a^{\beta} & 0 & 0 & a^{\beta} & 0 & 0 & b^{\beta} & 0 & 0 & 0 & b^{\beta} & 0 & 0 \\
0 & b^{\beta} & -c^{\beta} & 0 & 0 & 0 & 0 & b^{\beta} & 0 & -c^{\beta} & 0 & 0 & 0 \\
0 & 0 & 0 & 0 & 0 & 0 & 0 & b^{\beta} & c^{\beta} & 0 & 0 & 0 & 0 \\
0 & -b^{\beta} & c^{\beta} & 0 & 0 & 0 & 0 & -b^{\beta} & 0 & c^{\beta} & 0 & 0 & 0 \\
0 & 0 & 0 & 0 & 0 & 0 & 0 & 0 & 0 & c^{\beta} & 0 & 0 & 0 \\
0 & -b^{\beta} & c^{\beta} & 0 & 0 & 0 & 0 & -b^{\beta} & 0 & c^{\beta} & 0 & 0 & 0 \\
0 & b^{\beta} & -c^{\beta} & 0 & 0 & 0 & 0 & b^{\beta} & 0 & -c^{\beta} & 0 & 0 & 0
\end{array}\right] .
$$

The relation between the characteristic polynomials of the matrices $V_{\beta}, Q_{\beta}$, and $K_{\beta}$ is

$$
\begin{aligned}
P_{V_{\beta}}(t)= & \left(1-\left(2 a^{\beta}+b^{\beta}+c^{\beta}\right) t+\left(2(a c)^{\beta}-(b c)^{\beta}\right) t^{2}+\left(4(a b c)^{\beta}+\left(b^{2} c\right)^{\beta}\right) t^{3}\right. \\
& \left.+\left(b c^{2}\right)^{\beta} t^{3}+\left(2\left(a b^{2} c\right)^{\beta}-2\left(a b c^{2}\right)^{\beta}\right) t^{4}-2\left(a b^{2} c^{2}\right)^{\beta} t^{5}\right) \\
& \times\left(1-a^{\beta} t\right)\left(1+a^{\beta} t\right)\left(1-b^{\beta} t\right)\left(1-c^{\beta} t\right) \\
= & P_{Q_{\beta}}(t) P_{K_{\beta}}(t) .
\end{aligned}
$$

Then we can verify the statements of Theorems 3.5 and 3.6. By Theorem 1.1, we have

$$
\operatorname{dim}_{H}(E)=0.91994 \cdots ; \quad \gamma=0.0877769 \cdots ; \quad h_{\text {top }}=1.11531 \cdots .
$$

ACKNOWLEDGMENTS. The first author is partially supported by PRODEP, Medida 5, Acção 5.3. Both authors thank FCT (Portugal) for having in part supported this work through program POCTI. We are grateful to Nuno Martins for kindly reviewing this paper and useful comments.

\section{REFERENCES}

[1] V. Baladi and D. Ruelle, An extension of the theorem of Milnor and Thurston on the zeta functions of interval maps, Ergodic Theory Dynam. Systems 14 (1994), no. 4, 621632.

[2] R. Bowen and O. E. Lanford III., Zeta functions of restrictions of the shift transformation, Global Analysis (Berkeley, Calif, 1968), Proc. Sympos. Pure Math., vol. 14, American Mathematical Society, Rhode Island, 1970, pp. 43-49.

[3] A. Boyarsky and P. Góra, Laws of Chaos. Invariant Measures and Dynamical Systems in One Dimension, Probability and Its Applications, Birkhäuser Boston, Massachusetts, 1997.

[4] K. Falconer, Techniques in Fractal Geometry, John Wiley \& Sons, Chichester, 1997.

[5] S. Lang, Algebra, Graduate Texts in Mathematics, vol. 211, Springer-Verlag, New York, 2002.

[6] J. Milnor and W. Thurston, On iterated maps of the interval, Dynamical Systems (College Park, Md, 1986-87) (J. C. Alexander, ed.), Lecture Notes in Math., vol. 1342, SpringerVerlag, Berlin, 1988, pp. 465-563. 
[7] M. Mori, Fredholm determinant for piecewise linear transformations, Osaka J. Math. 27 (1990), no. 1, 81-116.

[8] J. L. Rocha and J. S. Ramos, Computing conditional measures and escape rates, submitted to Dynam. Systems Appl.

[9] _ On iterated maps of the interval with holes, J. Differ. Equations Appl. 9 (2003), no. 3-4, 319-335.

[10] _ IFS with overlaps and Hausdorff dimension, Grazer Math. Ber. 346 (2004), 355-376.

[11] D. Ruelle, Thermodynamic Formalism, Encyclopedia of Mathematics and Its Applications, vol. 5, Addison-Wesley Publishing, Massachusetts, 1978.

[12] _ Thermodynamic formalism for maps satisfying positive expansiveness and specification, Nonlinearity 5 (1992), no. 6, 1223-1236.

[13] _ _ Dynamical Zeta Functions for Piecewise Monotone Maps of the Interval, CRM Monograph Series, vol. 4, American Mathematical Society, Rhode Island, 1994.

[14] H. van den Bedem and N. Chernov, Expanding maps of an interval with holes, Ergodic Theory Dynam. Systems 22 (2002), no. 3, 637-654.

[15] P. Walters, An Introduction to Ergodic Theory, Graduate Texts in Mathematics, vol. 79, Springer-Verlag, New York, 1982.

J. Leonel Rocha: Seç̧ão de Matemática, Departamento de Engenharia Química, Instituto Superior de Engenharia de Lisboa, Rua Conselheiro Emídio Navarro, 1949-014 Lisboa, Portugal

E-mail address: jrocha@math. ist.ut1.pt

J. Sousa Ramos: Departamento de Matemática, Instituto Superior Técnico, avenida Rovisco Pais 1, 1049-001 Lisboa, Portugal

E-mail address: sramos@math.ist.ut1.pt 


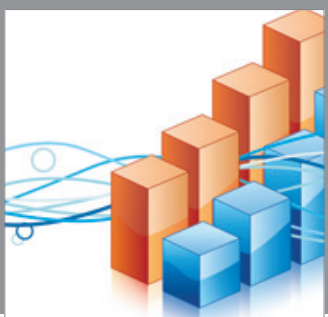

Advances in

Operations Research

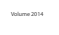

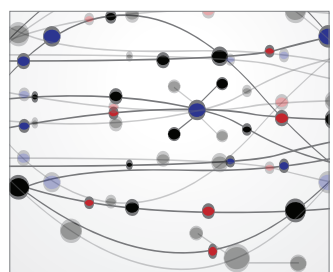

\section{The Scientific} World Journal
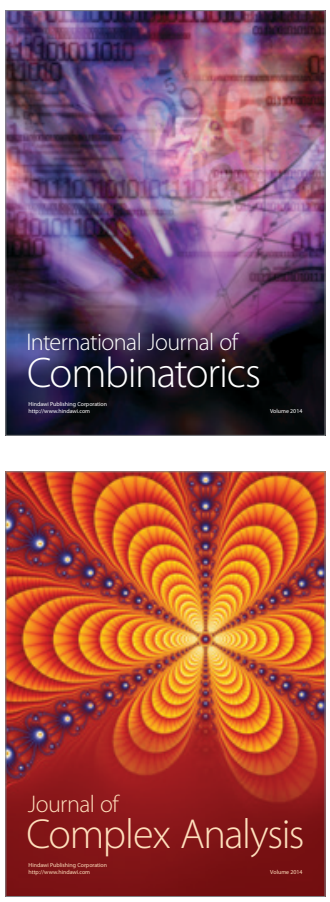

International Journal of

Mathematics and

Mathematical

Sciences
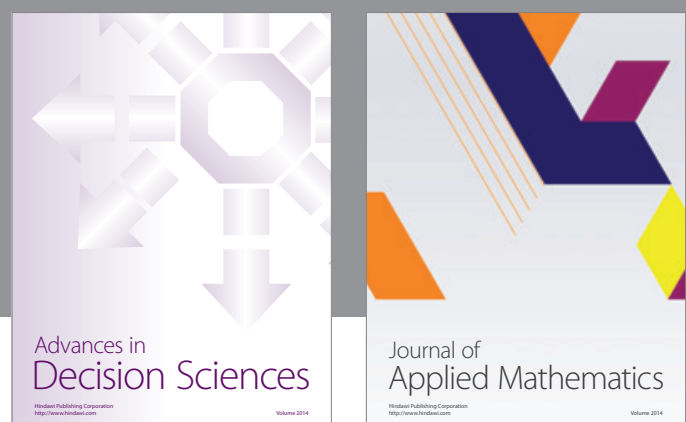

Journal of

Applied Mathematics
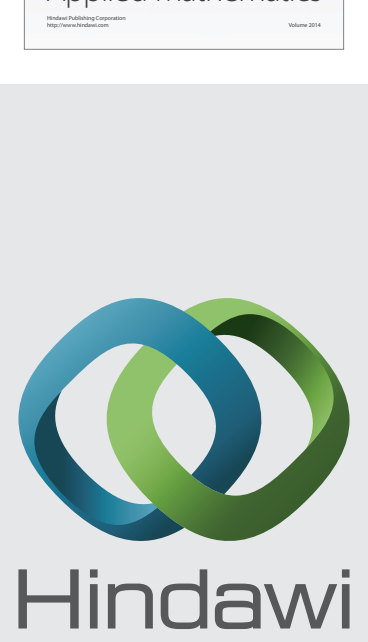

Submit your manuscripts at http://www.hindawi.com
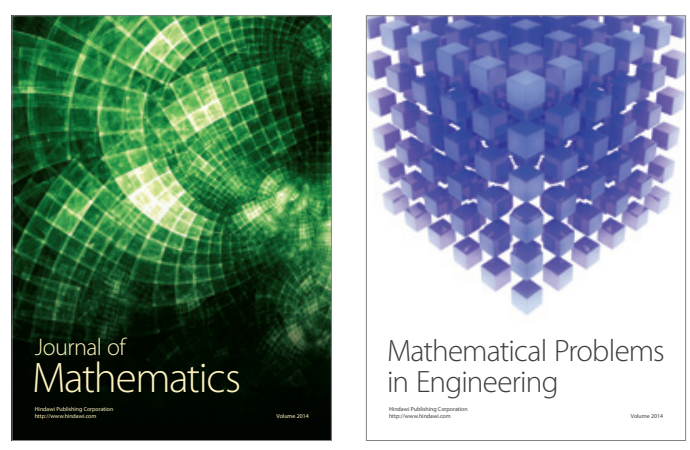

Mathematical Problems in Engineering
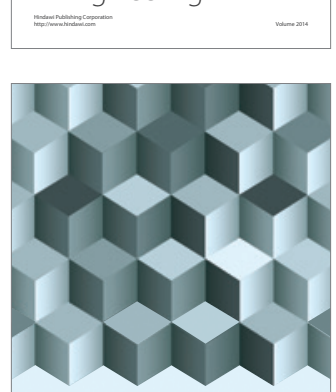

Journal of

Function Spaces
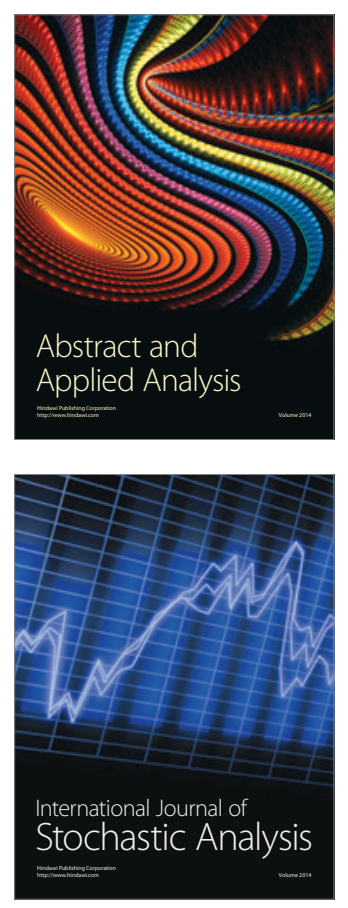

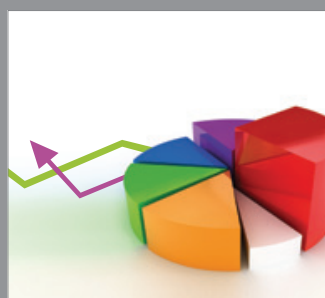

ournal of

Probability and Statistics

Promensencen
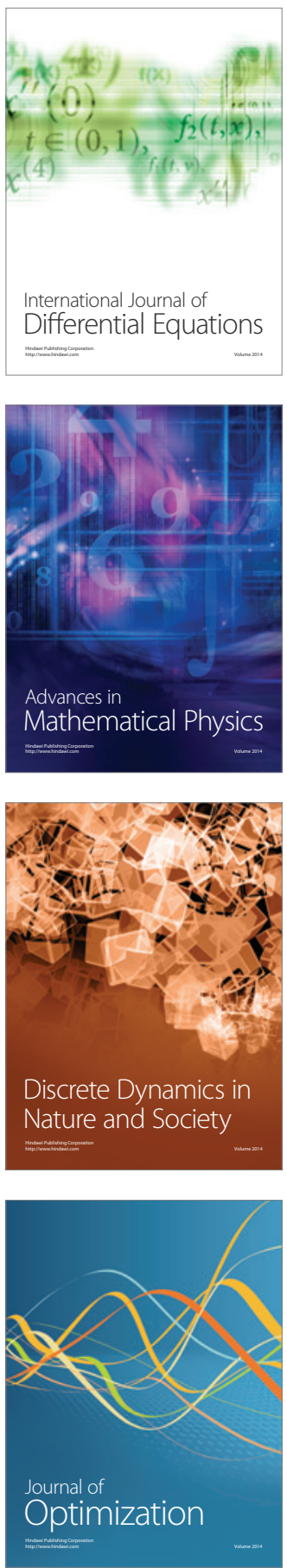